\title{
Impact of pollution on macroinvertebrates biodiversity in Ismailia Canal, Egypt
}

\author{
Magdy T. Khalil ${ }^{1}$, Amany S. Amer ${ }^{2}$, Montaser M. Sayed ${ }^{1}$ and Marian G. Nassif ${ }^{2}$ \\ 1- Faculty of Science, Ain Shams University, Cairo, Egypt. \\ 2- Central Laboratory for Environmental Quality Monitoring (CLEQM), National \\ Water Research Center (NWRC), Cairo, Egypt.
}

\section{ABSTRACT}

This study was conducted to monitor the adverse effect of the drainage wastewater of three main factories on the macroinvertebrates biodiversity of Ismailia Canal. Water and benthos samples were collected seasonally during 2010 from 10 stations along Ismailia Canal from El-Mezalat to Abu Zaabal city.

The results revealed that most of the physico-chemical parameters were within the permissible limits, although, Cairo Power station and Abu Zaabal factory are causing partial pollution. The community of macrobenthic invertebrates was represented by Mollusca (35.20 \%), Annelida (33.62 \%) and Arthropoda (31.18 \%). Limnodrilus hoffmeisteri and Chironomus larvae were dominating the whole studied area (32\% and $28.51 \%$ of total macrobenthos, respectively) and they are considered to be potential bioindicators for polluted ecosystem. Low species diversity and occurrence of pollution-tolerant species (e.g. Limnodrilus hoffmeisteri and Chironomus larvae) indicate that the water quality of the canal is deteriorated. A regular program for biomonitoring is recommended which will allow future changes and enhancing in this important ecosystem.

Key words: Ismailia Canal, macroinvertebrates, biodiversity, pollution

\section{INTRODUCTION}

Ismailia canal is one of the most important Nile tributaries in Egypt. It supplies water for a great number of the Egyptian citizens (about 12 million inhabitants), including those living in northern part of Cairo, Shubra El-Kheima, Mattaria, Musturod, Abu-Zaabal, Inchas, Belbeis, Abbasa, Abu-Hammad, Zagazeeg and El-Tell El-Kabier, before entering the Suez Canal Province (Fig. 1). It extends for about 128 $\mathrm{km}$ with $2.1 \mathrm{~m}$ depth and $18 \mathrm{~m}$ length. It is worthy to note that some factories are constructed at this area, discharging its wastes into the canal, which cause dramatic changes in the canal's water quality. Measuring of specific physico-chemical parameters in the contaminated aquatic ecosystem is important in determining the potential toxicity and its effects on the living organisms inhabiting that environment (Wrona and Cash, 1996). Most organisms living in a water body are sensitive to any change in their environment. Different organisms respond in different ways; the most extreme responses include death or migration to another habitat. Less obvious responses include reduced reproductive capacity and inhibition of certain enzyme system necessary for normal metabolism. Once the responses of particular aquatic organisms to a given change have been identified, they may be used to determine the quality of water with respect to its suitability for aquatic life (Chapman, 1992). The use of bioindicators in water quality assessment started more than a century ago. Macrobenthos are the most commonly organisms used as bioindicators because they are typically less mobile than fish; therefore, they provide a more localized assessment of their response to stream conditions (Barbour et al., 1999). 
Unfortunately, no detailed studies have been conducted yet on Ismailia Canal using macrobenthos as bioindicators. Therefore, this study was devoted to determine the impact of different kinds of pollutants on macroinvertebrates species composition and biodiversity in this important ecosystem.

\section{MATERIAL AND METHODS}

\section{Study area:}

Ten stations had been selected along Ismailia Canal extended from El-Mezalat to Abu Zaabal City. These stations are represented in Fig. (2):

Station 1: Inlet of the Canal.

Station 2: Before Cairo Electrical Company.

Station 3: Discharging Point of Cairo Electrical Company.

Station 4: After Cairo Electrical Company.

Station 5: Before Misr Petroleum Company.

Station 6: Discharging point of Misr Petroleum Company

Station 7: After Misr Petroleum Company.

Station 8: Before Abu Zaabal fertilizer and chemical Company.

Station 9: Discharging point of Abu Zaabal fertilizer and chemical Company.

Station 10: After Abu Zaabal fertilizer and chemical Company.

Water and benthos samples were collected seasonally during 2010. The water samples were collected from the subsurface layer of the canal using plastic containers. The collected water samples were kept in well stoppered polyethylene plastic bottles for most chemical analyses. Samples collected for heavy metals and cations were preserved by adding concentrated nitric acid to lower the $\mathrm{pH}$ to $<2$ to be protected against microbial reactions. All samples were transferred immediately in an ice-box to the laboratory for analysis. Water temperature, EC, pH, salinity, TDS as well as DO were measured in the field using multi-probe portable meter (WTW Model Oxi 197). Water transparency was measured using a standard Secchi disc of $25 \mathrm{~cm}$ diameter and the results were expressed in $\mathrm{cm}$ at the distance in which the Secchi disc disappeared. Carbonate and bicarbonate were measured by direct titration against $0.02 \mathrm{~N} \mathrm{HCl}$ using phenol phthaline and methyl red as indicators. Biological Oxygen Demand (BOD) was determined by Manometric BOD Oxitop system; dark brown bottle was filled with $426 \mathrm{ml}$ of water sample, supplied with magnetic stirrer and digital monitor on the opening of the bottle, and incubated at $35^{\circ} \mathrm{C}$ for 5 days. Ortho-phosphorus was determined by Stannous chloride method using spectrophotometer HACH (DR2400). Chloride, nitrate, nitrite, phosphate and sulfate concentrations in each sample were determined by Ion Chromatography (IC system-METROHM). Calcium and magnesium concentration in each sample was determined by Inductively Coupled Plasma-Optical Emission Spectroscopy (ICP-OES), (Perkin Elmer Optima-3000 Redial). Aluminum, arsenic, barium, cobalt, chromium, copper, iron, Molybdenum, lead, antimony, selenium and zinc concentrations in each water sample was determined by (ICP-OES), (Perkin Elmer Optima-3000 Redial).

The bottom fauna samples were collected by a square Ekman grab sampler with $225 \mathrm{~cm}^{2}$ opening area. Each sample was washed immediately to remove any adhering sediments or mud, using 500 $\mu \mathrm{m}$ mesh net and stored in plastic containers with $5 \%$ formalin as a preservative. In the laboratory, the samples were washed and sieved again through a net with $500 \mu \mathrm{m}$ mesh size. Benthic animals were sorted and identified to genera and species level using a zoom stereo microscope. Each group was counted and preserved in a glass bottle with $7 \%$ formalin. Identification of the collected 
species were carried out according to Brinkhurst (1971), Brown (1980), Ruffo (1982), Habashy (1993), El-Shemy (1994) and Ibrahim et al. (1999).

Species diversity of bottom fauna was calculated and evaluated to assess the impact of pollution on the degradation of species diversity, food chains and eventually the ecosystem using a computer soft ware namely Primer 5 version 5.2.0. The Similarity between the communities of macroinvertebrates in the ten stations of the canal was determined using Bray-Curtiz similarity index.

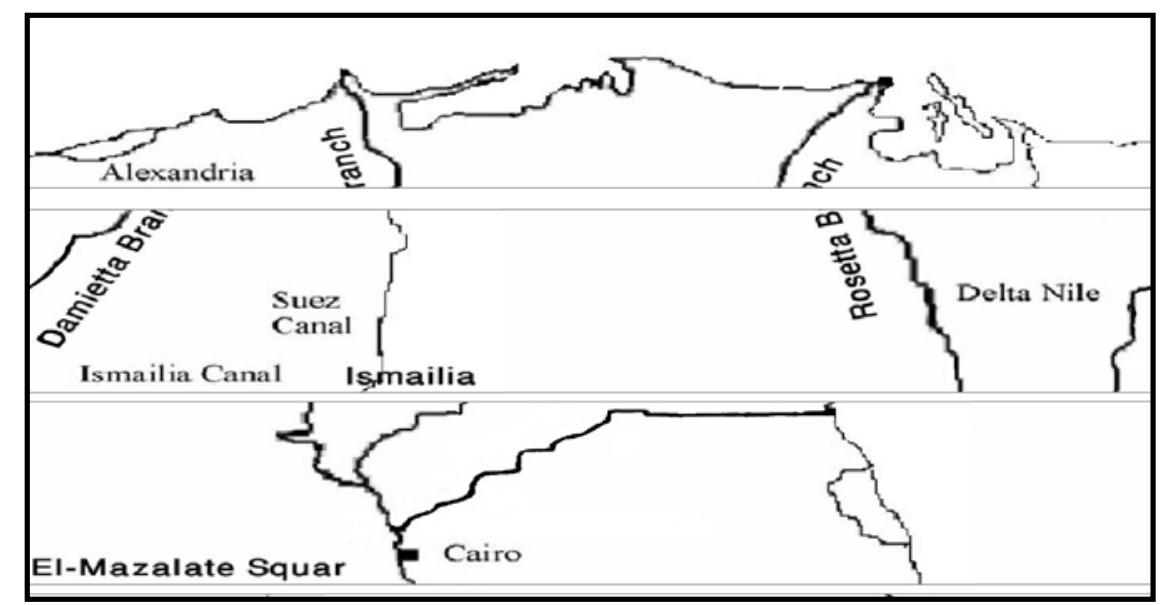

Fig.1: General view of Ismailia Canal.

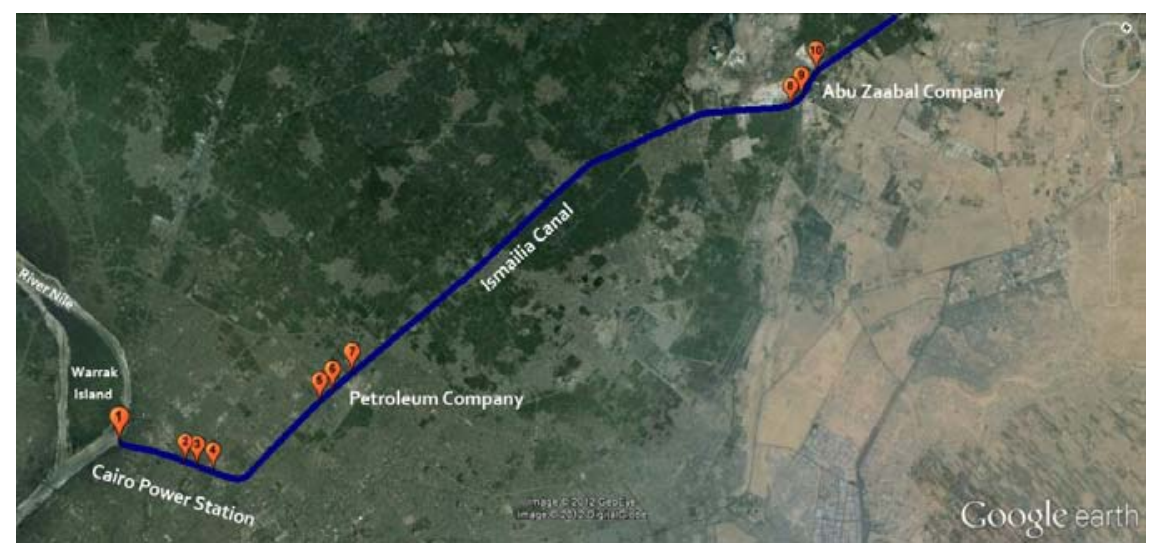

Fig. 2: A map showing Ismailia Canal sampling stations.

\section{RESULTS AND DISCUSSION}

\section{Physico-chemical Parameters: \\ Water temperature:}

As shown in Table (1), the maximum water temperature was recorded at station 6 with an annual average of $28.53{ }^{\circ} \mathrm{C}$, while the minimum was recorded at station 1 with an annual average of $22.95{ }^{\circ} \mathrm{C}$. This result is in accordance with Abdo et al. (2010) who reported a relative increase of the water temperature in front of the petroleum company causing thermal pollution. Regarding seasonal variation, summer exhibited the highest water temperature with an average of $31.6^{\circ} \mathrm{C}$, while winter exhibited the lowest with an average of $17.78{ }^{\circ} \mathrm{C}$. The low water temperature during winter and autumn is due to frequent clouds, high humidity, high current velocity, while high water temperature during summer and spring is due to clear atmosphere and great solar radiation (Shama et al., 2011). The analysis of variance (ANOVA) revealed significant regional and seasonal variation $(F=10.99$ and 467.457, $p<0.05$, respectively). 
Table 1: Seasonal variation of water temperature $\left({ }^{\circ} \mathrm{C}\right)$ in Ismailia Canal during 2010.

\begin{tabular}{|c|c|c|c|c|c|}
\hline & Winter & Spring & Summer & Autumn & Annual average \\
\hline St.1 & 16.00 & 26.00 & 29.00 & 20.80 & 22.95 \\
\hline St.2 & 16.90 & 26.90 & 30.00 & 21.80 & 23.90 \\
\hline St.3 & 17.30 & 27.50 & 33.00 & 24.80 & 25.65 \\
\hline St.4 & 17.10 & 27.00 & 31.00 & 21.80 & 24.23 \\
\hline St.5 & 18.10 & 28.00 & 30.00 & 22.20 & 24.58 \\
\hline St.6 & 22.20 & 32.20 & 36.00 & 23.70 & 28.53 \\
\hline St.7 & 17.90 & 28.00 & 31.00 & 23.40 & 25.08 \\
\hline St.8 & 17.50 & 27.70 & 32.00 & 24.10 & 25.33 \\
\hline St.9 & 17.40 & 29.30 & 32.00 & 23.10 & 25.45 \\
\hline St.10 & 17.40 & 28.10 & 32.00 & 23.30 & 25.20 \\
\hline Average & 17.78 & 28.07 & 31.60 & 22.90 & 25.09 \\
\hline
\end{tabular}

\section{Water transparency:}

The clarity of a natural water body is a major determinant of the condition and productivity of the sustain (APHA, 1998). Table (2) shows that maximum water transparency was recorded at station $1(93.75 \mathrm{~cm})$, while the minimum was at station 8 $(27.50 \mathrm{~cm})$. This result revealed the adverse effect of the different companies' effluents along the canal. This result is in accordance with Abdo and El-Nasharty (2010). On the other hand, the lowest transparency values were recorded during spring (Average of $38.50 \mathrm{~cm}$ ) due to the flourish of phyto- and zooplankton during this season (Abdo et al., 2010 ; Wissa, 2012). ANOVA test showed significant regional and seasonal variation $(F=3.15$ and $F=10.317, p<0.05$ respectively).

Table 2: Seasonal variation of water transparency (cm) in Ismailia Canal during 2010.

\begin{tabular}{|c|c|c|c|c|c|}
\hline & Winter & Spring & Summer & Autumn & Annual Average \\
\hline St.1 & 85.00 & 60.00 & 170.00 & 60.00 & 93.75 \\
\hline St.2 & 70.00 & 50.00 & 100.00 & 65.00 & 71.25 \\
\hline St.3 & 60.00 & 50.00 & 140.00 & 60.00 & 77.50 \\
\hline St.4 & 70.00 & 40.00 & 90.00 & 70.00 & 67.50 \\
\hline St.5 & 85.00 & 60.00 & 70.00 & 65.00 & 70.00 \\
\hline St.6 & 50.00 & 30.00 & 50.00 & 50.00 & 45.00 \\
\hline St.7 & 50.00 & 30.00 & 50.00 & 50.00 & 45.00 \\
\hline St.8 & 30.00 & 25.00 & 30.00 & 25.00 & 27.50 \\
\hline St.9 & 20.00 & 20.00 & 75.00 & 50.00 & 41.25 \\
\hline St.10 & 40.00 & 20.00 & 150.00 & 45.00 & 63.75 \\
\hline Average & 56.00 & 38.50 & 92.50 & 54.00 & 60.25 \\
\hline
\end{tabular}

\section{Electrical conductivity (EC):}

During the period of investigation, the EC values showed two peaks at stations 9 and 6 (505.75 and $503.50 \mu$ mohs, respectively), while the lowest $(428.50 \mu$ mohs) was recorded at station 2 (Table 3). These results may be attributed to the effluents of Abu Zaabal Company and the petroleum companies. This agreed with the results of Mohammed (2008) and Abdo et al. (2010) who recorded maximum EC values in front of the petroleum companies which are rich in heavy metals and organic matters. Furthermore, Abdo and El-Nasharty (2010) recorded the maximum EC values at Abu Zaabal Company area and the highest EC average value $(684.10 \mu$ mohs) was recorded during autumn which is in agree with Mohammed (2008). On the other hand, the lowest (343.50 $\mu$ mohs) was recorded during summer. Regarding ANOVA test, there was significant seasonal variation $(\mathrm{F}=103.862, \mathrm{P}<0.05)$. 
Table 3: Seasonal variation of water electrical conductivity ( $\mu$ moh) in Ismailia Canal during 2010.

\begin{tabular}{|c|c|c|c|c|c|}
\hline & Winter & Spring & Summer & Autumn & Annual Average \\
\hline St.1 & 435.00 & 354.00 & 325.00 & 784.00 & 474.50 \\
\hline St.2 & 439.00 & 364.00 & 334.00 & 577.00 & 428.50 \\
\hline St.3 & 439.00 & 351.00 & 335.00 & 650.00 & 443.75 \\
\hline St.4 & 444.00 & 350.00 & 334.00 & 640.00 & 442.00 \\
\hline St.5 & 440.00 & 353.00 & 334.00 & 614.00 & 435.25 \\
\hline St.6 & 488.00 & 405.00 & 430.00 & 691.00 & 503.50 \\
\hline St.7 & 443.00 & 353.00 & 329.00 & 860.00 & 496.25 \\
\hline St.8 & 446.00 & 355.00 & 335.00 & 600.00 & 434.00 \\
\hline St.9 & 464.00 & 468.00 & 341.00 & 750.00 & 505.75 \\
\hline St.10 & 456.00 & 358.00 & 338.00 & 675.00 & 456.75 \\
\hline Average & 449.40 & 371.10 & 343.50 & 684.10 & 462.03 \\
\hline
\end{tabular}

\section{Hydrogen Ion Concentration (pH):}

Although pH usually has no direct impact on consumers, it is one of the most important operational water quality parameters (WHO, 2003). During the present study, $\mathrm{pH}$ values were recorded at alkaline side (Table 4). The highest $\mathrm{pH}$ average value (8.49) was recorded at station 6 , while the lowest (7.63) was at station 9. These results explain the effects of the petroleum company and Abu Zaabal company's discharges on Ismailia Canal water quality. The results are also confirmed by Mohammed (2008) and Abdo and El-Nasharty (2010) who recorded the lowest pH value in front of Abu Zaabal Fertilizer Company. However, according to WHO (1993) the desirable $\mathrm{pH}$ for fresh water is in the range of 6.5-9.2. Thus, Ismailia Canal water still in the desirable $\mathrm{pH}$ ranges of fresh water. Regarding seasonal variation, the lowest $\mathrm{pH}$ value (7.88) was recorded during summer. This result is in coincident with Fishar (1999), Mohammed (2008) and Toufeek and Korium (2009). This could be explained on the basis of organic matter decomposition and releasing acidic gases (Elewa et al., 2007). On the other hand, the highest $\mathrm{pH}$ value (8.55) was during autumn. This may be attributed to the increase of photosynthetic activity (Yousry, 2009). The $\mathrm{pH}$ values were significantly correlated with the values of EC, DO, TDS, TSS and orthophosphate $(r=0.54,0.44,0.50,-0.52$ and -0.40 , respectively at $\mathrm{p}<0.05)$. ANOVA test revealed significant regional and seasonal variation $(\mathrm{F}=\mathrm{F}=2.95$ and $\mathrm{F}=$ 10.039, $\mathrm{p}<0.05$ respectively).

Table 4: Seasonal variation of pH in Ismailia Canal during 2010.

\begin{tabular}{|c|c|c|c|c|c|}
\hline & Winter & Spring & Summer & Autumn & Annual Average \\
\hline St.1 & 8.50 & 8.22 & 8.03 & 8.94 & 8.42 \\
\hline St.2 & 8.07 & 8.16 & 7.61 & 8.90 & 8.19 \\
\hline St.3 & 8.16 & 8.04 & 8.01 & 8.83 & 8.26 \\
\hline St.4 & 7.88 & 8.09 & 7.80 & 8.72 & 8.12 \\
\hline St.5 & 7.77 & 7.90 & 8.29 & 8.70 & 8.17 \\
\hline St.6 & 8.86 & 7.77 & 8.70 & 8.62 & 8.49 \\
\hline St.7 & 8.04 & 7.95 & 7.58 & 8.11 & 7.92 \\
\hline St.8 & 8.83 & 7.93 & 7.80 & 8.04 & 8.15 \\
\hline St.9 & 7.29 & 7.52 & 7.42 & 8.27 & 7.63 \\
\hline St.10 & 7.68 & 7.66 & 7.56 & 8.40 & 7.83 \\
\hline Average & 8.11 & 7.92 & 7.88 & 8.55 & 8.12 \\
\hline
\end{tabular}




\section{Dissolved Oxygen (DO):}

Dissolved oxygen is a very important factor to the aquatic organisms, because it affects their biological processes, respiration and oxidation of the organic matter in water and sediments (Abdo et al., 2010). During the present study, the results showed that the highest DO average value $(10.69 \mathrm{mg} / \mathrm{l})$ was recorded at station 9 , while the lowest $(7.12 \mathrm{mg} / \mathrm{l})$ was recorded at stations 2 (Table 5). It was noticeable that station 9 exhibits the highest DO value $(25.00 \mathrm{mg} / \mathrm{l})$ during autumn. This result may be attributed to the mixing effect of Abu Zaabal effluents which increase the solubility of the atmospheric oxygen gas. On the other hand, autumn exhibited the maximum DO value with an average of $16.33 \mathrm{mg} / \mathrm{l}$, which could be attributed to the prevailing wind action that permits increased solubility of atmospheric oxygen gas (Ibrahim et al., 2008). Although, summer exhibited the lowest values with an average of $4.15 \mathrm{mg} / \mathrm{l}$, which might be due to the elevation of water temperature and the increase in oxidative processes of organic matter which lead to oxygen depletion (Abdel-Satar and Elewa, 2001; Abdel-Satar, 2005; Elewa et al., 2007; Hassan, 2008). ANOVA test revealed significant seasonal variation $(F=80.700, p<0.05)$.

Table 5: Seasonal variation of Dissolved Oxygen DO (mg/l) in Ismailia Canal during 2010.

\begin{tabular}{|c|c|c|c|c|c|}
\hline & Winter & Spring & Summer & Autumn & $\begin{array}{c}\text { Annual } \\
\text { Average }\end{array}$ \\
\hline St.1 & 6.10 & 7.26 & 4.00 & 13.52 & 7.72 \\
\hline St.2 & 5.90 & 6.30 & 3.70 & 12.57 & 7.12 \\
\hline St.3 & 5.74 & 7.22 & 4.02 & 16.58 & 8.39 \\
\hline St.4 & 5.67 & 9.59 & 3.70 & 14.60 & 8.39 \\
\hline St.5 & 5.69 & 7.54 & 3.85 & 12.40 & 7.37 \\
\hline St.6 & 5.70 & 8.57 & 4.35 & 17.26 & 8.97 \\
\hline St.7 & 6.40 & 8.60 & 4.28 & 17.42 & 9.18 \\
\hline St.8 & 5.93 & 8.71 & 4.52 & 15.00 & 8.54 \\
\hline St.9 & 5.91 & 7.00 & 4.84 & 25.00 & 10.69 \\
\hline St.10 & 5.59 & 8.59 & 4.20 & 18.90 & 9.32 \\
\hline Average & 5.86 & 7.94 & 4.15 & 16.33 & 8.57 \\
\hline
\end{tabular}

\section{Biological Oxygen Demand (BOD):}

The maximum average value $(8.25 \mathrm{mg} / \mathrm{l})$ was recorded at station 6 , while stations 3 and 4 exhibited the minimum average values $(3.50$ and $3.00 \mathrm{mg} / \mathrm{l}$, respectively) (Table 6). It was noticeable that station 6 exhibits the highest BOD value during winter $(15.00 \mathrm{mg} / \mathrm{l})$. This is attributed to the negative impact of the high amount of petroleum byproducts discharged from the petroleum companies into the canal. According to Hassan (2008) the biological oxygen demand (BOD) is directly related to the decomposition of organic matter found in the water and hence the high values of BOD reflect the degree of pollution in the studied habitat. Hence, the BOD values were within the permissible limits $(6 \mathrm{mg} / \mathrm{l}$ according to the Egyptian law 48/1982) except at station 6 (average of $8.25 \mathrm{mg} / \mathrm{l}$ ) which might pollute the canal. Seasonally, the values of BOD ranged between 2-15, 2-5, 4-7 and 3-9 mg/l during winter, spring, summer and autumn, respectively. The highest BOD average value (5.50 $\mathrm{mg} / \mathrm{l})$ was recorded during winter and this may be due to the decay of macrophytes and the high velocity and circulation of the water leading to uplifting the organic matter from the bottom to the surface (Fishar et al.,2006). Using ANOVA test, there were significant regional and seasonal variation $(\mathrm{p}<0.05)$. 
Table 6: Seasonal variation of BOD (mg/l) in Ismailia Canal during 2010.

\begin{tabular}{|c|c|c|c|c|c|}
\hline & Winter & Spring & Summer & Autumn & Annual Average \\
\hline St.1 & 5.00 & 3.00 & 5.00 & 4.00 & 4.25 \\
\hline St.2 & 2.00 & 5.00 & 5.00 & 3.00 & 3.75 \\
\hline St.3 & 4.00 & 2.00 & 5.00 & 3.00 & 3.50 \\
\hline St.4 & 3.00 & 2.00 & 4.00 & 3.00 & 3.00 \\
\hline St.5 & 5.00 & 2.00 & 5.00 & 5.00 & 4.25 \\
\hline St.6 & 15.00 & 4.00 & 5.00 & 9.00 & 8.25 \\
\hline St.7 & 7.00 & 2.00 & 5.00 & 4.00 & 4.50 \\
\hline St.8 & 6.00 & 4.00 & 7.00 & 8.00 & 6.25 \\
\hline St.9 & 4.00 & 2.00 & 7.00 & 4.00 & 4.25 \\
\hline St.10 & 4.00 & 5.00 & 4.00 & 5.00 & 4.50 \\
\hline Average & 5.50 & 3.10 & 5.20 & 4.80 & 4.65 \\
\hline
\end{tabular}

\section{Carbonate and bicarbonate alkalinity:}

Carbonate was not detected at all during the period of study, while bicarbonate values are represented in Table (7). ANOVA test exhibited significant variation only in the seasons with lowest values during summer due to the high evaporation rate in summer season (Mohammed, 2008).

Table 7: Seasonal variation of bicarbonates (mg/l) in studied area of Ismailia Canal during 2010.

\begin{tabular}{|c|c|c|c|c|c|}
\hline & Winter & Spring & Summer & Autumn & Annual Average \\
\hline St.1 & 180.00 & 190.00 & 153.00 & 180.00 & 175.75 \\
\hline St.2 & 175.00 & 200.00 & 148.00 & 175.00 & 174.50 \\
\hline St.3 & 173.00 & 189.00 & 156.00 & 182.00 & 175.00 \\
\hline St.4 & 170.00 & 199.00 & 158.00 & 185.00 & 178.00 \\
\hline St.5 & 190.00 & 188.00 & 165.00 & 180.00 & 180.75 \\
\hline St.6 & 175.00 & 190.00 & 146.00 & 190.00 & 175.25 \\
\hline St.7 & 173.00 & 198.00 & 145.00 & 170.00 & 171.50 \\
\hline St.8 & 170.00 & 175.00 & 151.00 & 195.00 & 172.75 \\
\hline St.9 & 168.00 & 204.00 & 147.00 & 175.00 & 173.50 \\
\hline St.10 & 178.00 & 192.00 & 153.00 & 178.00 & 175.25 \\
\hline Average & 175.20 & 192.50 & 152.20 & 181.00 & 175.23 \\
\hline
\end{tabular}

\section{Nutrients:}

Nitrite was detected only during winter with lower values than the corresponding values of nitrate due to the fast conversion of $\mathrm{NO}_{2}{ }^{-}$to $\mathrm{NO}_{3}{ }^{-}$ions by nitrifying bacteria (Shama et al., 2011). The increase in $\mathrm{NO}_{2}{ }^{-}$during cold seasons might be attributed to low consumption by phytoplankton as well as the oxidation of ammonia by nitrifying bacteria and biological nitrification (Rabeh, 2000 and Abdo et al., 2010) (Table 8). In the same manner, Nitrate recorded its maximum values during winter $(6.90 \mathrm{mg} / \mathrm{l})$ and the minimum values during summer $(0.03 \mathrm{mg} / \mathrm{l})$ (Table 9$)$, which might be attributed to the uptake of nitrate by natural phytoplankton as well as the effect of denitrifying bacteria (Saad and Abdel-Moati, 1997; Sabae and AbdelSatar, 2001). On the other hand, it was noticeable that station 6 was higher than all the other stations during winter (13.76 mg/l), which is mainly attributed to the effluents discharge of petroleum companies at this area (Abdo,1998; Sabae et al., 2006 and Abdo et al., 2010). Regarding orthophosphate, as the main reason for eutrophication, the minimal average value $(0.04 \mathrm{mg} / \mathrm{l})$ was recorded at station 1 , while the maximal average value was $(0.61 \mathrm{mg} / \mathrm{l})$ at station 9 with the highest value at station 9 during 
spring (1.38 mg/l) as a result of the discharges from Abu Zaabal Company for Fertilizers (Table 10).

Table 8: Seasonal variation of nitrite nitrogen $\left(\mathrm{NO}_{2}^{-}\right)(\mathrm{mg} / \mathrm{l})$ in Ismailia Canal during 2010.

\begin{tabular}{|c|c|c|c|c|c|}
\hline & Winter & Spring & Summer & Autumn & Annual Average \\
\hline St.1 & $<0.2$ & $<0.2$ & $<0.2$ & $<0.2$ & $<0.2$ \\
\hline St.2 & $<0.2$ & $<0.2$ & $<0.2$ & $<0.2$ & $<0.2$ \\
\hline St.3 & $<0.2$ & $<0.2$ & $<0.2$ & $<0.2$ & $<0.2$ \\
\hline St.4 & $<0.2$ & $<0.2$ & $<0.2$ & $<0.2$ & $<0.2$ \\
\hline St.5 & 0.78 & $<0.2$ & $<0.2$ & $<0.2$ & 0.2 \\
\hline St.6 & $<0.2$ & $<0.2$ & $<0.2$ & $<0.2$ & $<0.2$ \\
\hline St.7 & 0.74 & $<0.2$ & $<0.2$ & $<0.2$ & 0.19 \\
\hline St.8 & 0.58 & $<0.2$ & $<0.2$ & $<0.2$ & 0.145 \\
\hline St.9 & 0.47 & $<0.2$ & $<0.2$ & $<0.2$ & 0.117 \\
\hline St.10 & 1.27 & $<0.2$ & $<0.2$ & $<0.2$ & 0.31 \\
\hline Average & 3.84 & $<0.2$ & $<0.2$ & $<0.2$ & 0.96 \\
\hline
\end{tabular}

Table 9: Seasonal variation of Nitrate nitrogen $\left(-\mathrm{NO}_{3}{ }^{-}\right)(\mathrm{mg} / \mathrm{l})$ in Ismailia Canal during 2010.

\begin{tabular}{|c|c|c|c|c|c|}
\hline & Winter & Spring & Summer & Autumn & Annual Average \\
\hline St.1 & 4.88 & 4.20 & $<0.2$ & 0.26 & 2.34 \\
\hline St.2 & 9.37 & 9.60 & $<0.2$ & 0.36 & 4.83 \\
\hline St.3 & 4.96 & 4.20 & 0.33 & 0.27 & 2.44 \\
\hline St.4 & 5.45 & 4.30 & $<0.2$ & 0.61 & 2.59 \\
\hline St.5 & 6.23 & 3.50 & $<0.2$ & 0.20 & 2.48 \\
\hline St.6 & 13.76 & 5.30 & $<0.2$ & 0.26 & 4.83 \\
\hline St.7 & 8.64 & 3.80 & $<0.2$ & 0.21 & 3.16 \\
\hline St.8 & 6.38 & 5.10 & $<0.2$ & 0.20 & 2.92 \\
\hline St.9 & 3.90 & 5.90 & $<0.2$ & 0.26 & 2.52 \\
\hline St.10 & 5.48 & 4.40 & $<0.2$ & 0.76 & 2.66 \\
\hline Average & 6.90 & 5.03 & 0.03 & 0.34 & 3.08 \\
\hline
\end{tabular}

Table 10: Seasonal variation of orthophosphate (mg/l) in Ismailia Canal during 2010.

\begin{tabular}{|c|c|c|c|c|c|}
\hline & Winter & Spring & Summer & Autumn & Annual Average \\
\hline St.1 & 0.01 & 0.049 & 0.031 & 0.06 & 0.04 \\
\hline St.2 & 0.03 & 0.340 & 0.025 & 0.04 & 0.11 \\
\hline St.3 & 0.01 & 0.240 & 0.029 & 0.07 & 0.09 \\
\hline St.4 & 0.03 & 0.150 & 0.045 & 0.05 & 0.07 \\
\hline St.5 & 0.03 & 0.081 & 0.000 & 0.07 & 0.05 \\
\hline St.6 & 0.01 & 0.210 & 0.031 & 0.06 & 0.08 \\
\hline St.7 & 0.00 & 0.144 & 0.021 & 0.09 & 0.06 \\
\hline St.8 & 0.01 & 0.055 & 0.025 & 0.07 & 0.04 \\
\hline St.9 & 0.57 & 1.381 & 0.146 & 0.34 & 0.61 \\
\hline St.10 & 0.39 & 0.812 & 0.069 & 0.09 & 0.34 \\
\hline Average & 0.11 & 0.35 & 0.04 & 0.09 & 0.15 \\
\hline
\end{tabular}

\section{Total suspended solids (TSS) and total dissolved solids (TDS):}

The highest TSS average value $(28.25 \mathrm{mg} / \mathrm{l})$ was recorded at station 8 , while the lowest value $(6.00 \mathrm{mg} / \mathrm{l})$ at station 1 , with a peak value at station 8 during summer $(73.00 \mathrm{mg} / \mathrm{l})$. ANOVA test revealed only seasonal significant variation with maximum value $(26.80 \mathrm{mg} / \mathrm{l})$ during spring as a result of the high standing crop of zooplankton and blooming of phytoplankton (Saad, 1973; Abdel-Satar, 1998 \& 2005), while the lowest $(4.60 \mathrm{mg} / \mathrm{l})$ was during autumn (Table 11). On the other hand, the results of TDS showed significant regional variation $(\mathrm{F}=2.3$ and $\mathrm{p}<0.05)$, while the highest average value $(324.75 \mathrm{mg} / \mathrm{l})$ was recorded at station 6 and the lowest value 
(272.75 mg/l) at station 1 (Table 12). This is mainly attributed to the effluents of the Petroleum Company. In addition station 9 was higher than stations 8 and 10 which reflect the adverse effect of Abu Zaabal Fertilizer Company on Ismailia Canal. ANOVA test also exhibited significant seasonal variation of TDS values ( $F=103.76$, $\mathrm{p}<0.05)$ with a maximum average value $(417.30 \mathrm{mg} / \mathrm{l})$ during autumn, while the lowest (226.70 mg/l) was during summer due to the wind action which increases the solubility of solids during autumn.

Table11: Seasonal variation of TSS (mg/l) in Ismailia Canal during 2010.

\begin{tabular}{|c|c|c|c|c|c|}
\hline & Winter & Spring & Summer & Autumn & Annual Average \\
\hline St.1 & 5.00 & 17.00 & 1.00 & 1.00 & 6.00 \\
\hline St.2 & 19.00 & 20.00 & 1.00 & 2.00 & 10.50 \\
\hline St.3 & 7.00 & 36.00 & 1.00 & 4.00 & 12.00 \\
\hline St.4 & 6.00 & 22.00 & 9.00 & 2.00 & 9.75 \\
\hline St.5 & 7.00 & 33.00 & 15.00 & 4.00 & 14.75 \\
\hline St.6 & 1.00 & 27.00 & 7.00 & 2.00 & 9.25 \\
\hline St.7 & 9.00 & 18.00 & 42.00 & 4.00 & 18.25 \\
\hline St.8 & 10.00 & 23.00 & 73.00 & 7.00 & 28.25 \\
\hline St.9 & 21.00 & 36.00 & 16.00 & 10.00 & 20.75 \\
\hline St.10 & 32.00 & 36.00 & 17.00 & 10.00 & 23.75 \\
\hline Average & 11.70 & 26.80 & 18.20 & 4.60 & 15.33 \\
\hline
\end{tabular}

Table12: Seasonal variation of TDS (mg/l) in Ismailia Canal during 2010.

\begin{tabular}{|c|c|c|c|c|c|}
\hline & Winter & Spring & Summer & Autumn & Annual Average \\
\hline St.1 & 275.00 & 235.00 & 216.00 & 365.00 & 272.75 \\
\hline St.2 & 280.00 & 244.00 & 219.00 & 368.00 & 277.75 \\
\hline St.3 & 281.00 & 236.00 & 217.00 & 402.00 & 284.00 \\
\hline St.4 & 284.00 & 236.00 & 220.00 & 388.00 & 282.00 \\
\hline St.5 & 281.00 & 235.00 & 217.00 & 385.00 & 279.50 \\
\hline St.6 & 308.00 & 269.00 & 284.00 & 438.00 & 324.75 \\
\hline St.7 & 284.00 & 235.00 & 222.00 & 513.00 & 313.50 \\
\hline St.8 & 282.00 & 237.00 & 221.00 & 380.00 & 280.00 \\
\hline St.9 & 295.00 & 312.00 & 224.00 & 453.00 & 321.00 \\
\hline St.10 & 288.00 & 243.00 & 227.00 & 481.00 & 309.75 \\
\hline Average & 285.80 & 248.20 & 226.70 & 417.30 & 294.50 \\
\hline
\end{tabular}

\section{Heavy Metals:}

Lead, zinc, iron and copper showed the maximum values at station 9 with an average of $0.061 \mathrm{mg} / \mathrm{l}, 0.318 \mathrm{mg} / \mathrm{l}, 1.425 \mathrm{mg} / \mathrm{l}$ and $0.227 \mathrm{mg} / \mathrm{l}$, respectively (Table 13). Although, cobalt showed its maximum concentration at station 10 with an average of $0.014 \mathrm{mg} / \mathrm{l}$. Barium and chromium showed the maximum concentrations at station 5 with an average of $0.786 \mathrm{mg} / \mathrm{l}$ and $0.227 \mathrm{mg} / \mathrm{l}$, respectively. Aluminum exhibited the maximum value at station 6 with an average of $1.227 \mathrm{mg} / \mathrm{l}$. On the other hand, arsenic, antimony, selenium and molybdenum were not detected during the period of study. 
Table 13: Seasonal variation of the studied heavy metals in Ismailia Canal during 2010.

\begin{tabular}{|c|c|c|c|c|c|c|c|c|}
\hline & $\mathrm{Al}$ & $\mathrm{Cr}$ & $\mathrm{Ba}$ & $\mathrm{Cu}$ & Co & $\mathrm{Fe}$ & $\mathrm{Zn}$ & $\mathrm{Pb}$ \\
\hline \multicolumn{9}{|l|}{ Winter } \\
\hline St. 1 & 0.055 & 0.016 & 0 & 0 & 0 & 0 & 0.027 & 0 \\
\hline St. 2 & 0.055 & 0.01 & 0 & 0 & 0 & 0 & 0.018 & 0 \\
\hline St. 3 & 0.053 & 0.01 & 0 & 0.018 & 0 & 0 & 0.043 & 0 \\
\hline St. 4 & 0.028 & 0.007 & 0 & 0.026 & 0 & 0 & 0.029 & 0 \\
\hline St. 5 & 0.615 & 0.006 & 0 & 0 & 0 & 0 & 0.029 & 0 \\
\hline St. 6 & 0.548 & 0.003 & 0 & 0 & 0 & 0.028 & 0.026 & 0 \\
\hline St. 7 & 0.487 & 0.005 & 0 & 0 & 0 & 0.261 & 0.031 & 0 \\
\hline St. 8 & 0.202 & 0 & 0 & 0 & 0 & 0.297 & 0.037 & 0 \\
\hline St. 9 & 0.213 & 0.089 & 0.11 & 0.03 & 0 & 0.485 & 0.014 & 0 \\
\hline St. 10 & 0.444 & 0.069 & 0.11 & 0.022 & 0 & 0.48 & 0.02 & 0 \\
\hline \multicolumn{9}{|l|}{ Spring } \\
\hline St. 1 & 0.526 & 0.069 & 0.099 & 0.1 & 0 & 0.808 & 0.061 & 0.006 \\
\hline St. 2 & 0.735 & 0.079 & 0.03 & 0.12 & 0 & 1.326 & 0.292 & 0.009 \\
\hline St. 3 & 1.243 & 0.051 & 0.063 & 0.469 & 0 & 2.392 & 0.196 & 0.117 \\
\hline St. 4 & 0.779 & 0.081 & 0.009 & 0.195 & 0 & 1.301 & 0.082 & 0.004 \\
\hline St. 5 & 0.076 & 0.1 & 0 & 0.006 & 0 & 0.229 & 0.027 & 0 \\
\hline St. 6 & 0.639 & 0.053 & 0.018 & 0.58 & 0 & 2.608 & 0.8 & 0.166 \\
\hline St. 7 & 2.054 & 0.073 & 0.062 & 0.154 & 0 & 2.491 & 0.085 & 0.008 \\
\hline St. 8 & 1.113 & 0.081 & 0.021 & 0.022 & 0 & 1.239 & 0.042 & 0 \\
\hline St. 9 & 3.069 & 0.092 & 0.02 & 0.392 & 0 & 4.199 & 0.234 & 0.243 \\
\hline St. 10 & 1.69 & 0.1 & 0.031 & 0.252 & 0 & 2.156 & 0.121 & 0.003 \\
\hline \multicolumn{9}{|c|}{ Summer } \\
\hline St. 1 & 0.058 & 0.013 & 0.051 & 0.13 & 0 & 0.071 & 0.03 & 0.013 \\
\hline St. 2 & 0.035 & 0.074 & 0.05 & 0.349 & 0.03 & 0.925 & 0.079 & 0 \\
\hline St. 3 & 0.061 & 0.124 & 0.019 & 0.139 & 0 & 0.17 & 0.045 & 0.006 \\
\hline St. 4 & 0.056 & 0.099 & 0.046 & 0.228 & 0 & 0 & 0.055 & 0 \\
\hline St. 5 & 0.056 & 0.799 & 0.042 & 0.146 & 0.025 & 0 & 0.033 & 0.017 \\
\hline St. 6 & 0.058 & 0.062 & 0.021 & 0.179 & 0 & 0 & 0.025 & 0.018 \\
\hline St. 7 & 0.051 & 0.083 & 0.05 & 0.305 & 0.013 & 1.295 & 0.051 & 0.021 \\
\hline St. 8 & 0.038 & 0.207 & 0.057 & 0.155 & 0.027 & 0 & 0.033 & 0.024 \\
\hline St. 9 & 0.082 & 0.058 & 0.019 & 0.169 & 0.021 & 0 & 0.039 & 0 \\
\hline St. 10 & 0.058 & 0.088 & 0.053 & 0.172 & 0.031 & 0 & 0.035 & 0.017 \\
\hline \multicolumn{9}{|c|}{ Autumn } \\
\hline St. 1 & 0.213 & 0.007 & 1.946 & 0.132 & 0.016 & 0.734 & 0.327 & 0 \\
\hline St. 2 & 0.197 & 0.009 & 1.798 & 0.025 & 0.002 & 0.392 & 0.068 & 0 \\
\hline St. 3 & 0.393 & 0.004 & 2.275 & 0.043 & 0.001 & 0.527 & 0.144 & 0 \\
\hline St. 4 & 0.494 & 0.006 & 2.57 & 0.128 & 0.015 & 0.723 & 0.377 & 0 \\
\hline St. 5 & 2.132 & 0.004 & 3.101 & 0.136 & 0.017 & 0.656 & 0.391 & 0 \\
\hline St. 6 & 3.661 & 0.022 & 1.881 & 0.117 & 0.015 & 0.792 & 0.349 & 0 \\
\hline St. 7 & 1.56 & 0.01 & 2.232 & 0.07 & 0.007 & 0.721 & 0.222 & 0 \\
\hline St. 8 & 2.42 & 0.011 & 2.462 & 0.03 & 0.004 & 0.805 & 0.081 & 0 \\
\hline St. 9 & 1.517 & 0.026 & 2.218 & 0.317 & 0.019 & 1.016 & 0.985 & 0 \\
\hline St. 10 & 1.46 & 0.011 & 2.31 & 0.148 & 0.026 & 1.007 & 0.477 & 0 \\
\hline
\end{tabular}

\section{Macrobenthic fauna:}

\section{Population Density and Community Structure of Macrobenthic Fauna:}

Eighteen Macrobenthic species were identified in the present study. They were represented in three phyla namely؛ Mollusca, Annelida and Arthropoda (Fig. 3). Their annual percentages were $35.20 \%$, 33.62\% and 31.18\%, respectively. The total density 
of the macrobenthos was 376.83 ind. $/ \mathrm{m}^{2}$ (Table 14). Similar results were recorded by Wissa (2002) in the River Nile at North Cairo.

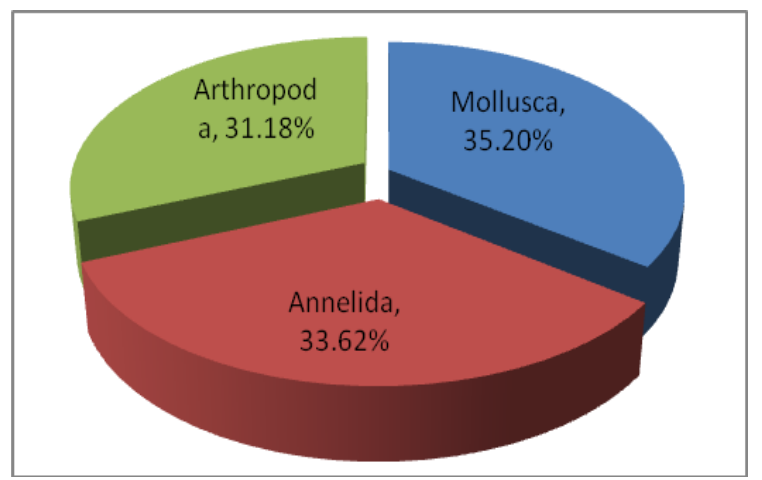

Fig. 3: Community structure of macrobenthos in Ismailia Canal during 2010.

Table 14: Seasonal species composition of macrobenthos in Ismailia Canal during 2010.

\begin{tabular}{|c|c|c|c|c|c|c|c|}
\hline Species & $\begin{array}{c}\text { Av. } \\
\text { Winter }\end{array}$ & $\begin{array}{c}\text { Av. } \\
\text { Spring }\end{array}$ & $\begin{array}{c}\text { Av. } \\
\text { Summer }\end{array}$ & $\begin{array}{c}\text { Av. } \\
\text { Autumn }\end{array}$ & Average & \%/Macro & \%/group \\
\hline \multicolumn{8}{|l|}{ Mollusca } \\
\hline Bellamya unicolor & 0.00 & 31.10 & 22.20 & 4.40 & 14.43 & $3.83 \%$ & $10.87 \%$ \\
\hline Corbicula consorbina & 17.50 & 6.60 & 15.50 & 2.20 & 10.45 & $2.77 \%$ & $7.88 \%$ \\
\hline Caelatura sp. & 12.50 & 2.20 & 4.40 & 4.40 & 5.88 & $1.56 \%$ & $4.43 \%$ \\
\hline Cleopatra sp. & 22.50 & 0.00 & 31.10 & 31.10 & 21.18 & $5.62 \%$ & $15.96 \%$ \\
\hline Lanistes carinatus & 0.00 & 4.40 & 0.00 & 0.00 & 1.10 & $0.29 \%$ & $0.83 \%$ \\
\hline Lymnaea sp. & 0.00 & 24.40 & 0.00 & 0.00 & 6.10 & $1.62 \%$ & $4.60 \%$ \\
\hline Melanoides tuberculata & 20.00 & 88.90 & 75.40 & 84.30 & 67.15 & $17.82 \%$ & $50.62 \%$ \\
\hline Physa acuta & 0.00 & 2.20 & 0.00 & 0.00 & 0.55 & $0.15 \%$ & $0.41 \%$ \\
\hline Bulinus truncatus & 0.00 & 6.70 & 0.00 & 0.00 & 1.68 & $0.44 \%$ & $1.26 \%$ \\
\hline Theodoxus niloticus & 10.00 & 2.20 & 2.20 & 2.20 & 4.15 & $1.10 \%$ & $1.13 \%$ \\
\hline Subtotal & 82.50 & 168.70 & 150.80 & 128.60 & 132.65 & $35.20 \%$ & $100.00 \%$ \\
\hline \multicolumn{8}{|l|}{ Annelida } \\
\hline Limnodrilus hoffmeisteri & 40.00 & 44.50 & 182.20 & 215.60 & 120.58 & $32.00 \%$ & $95.18 \%$ \\
\hline Helobdella confera & 0.00 & 4.40 & 0.00 & 0.00 & 1.10 & $0.29 \%$ & $0.87 \%$ \\
\hline Brachiura sowerbyi & 0.00 & 17.80 & 2.20 & 0.00 & 5.00 & $1.33 \%$ & $3.95 \%$ \\
\hline Subtotal & 40.00 & 66.70 & 184.40 & 215.60 & 126.68 & $33.62 \%$ & $100.00 \%$ \\
\hline \multicolumn{8}{|l|}{ Crustacea } \\
\hline Cardina nilotica & 15.00 & 11.10 & 0.00 & 0.00 & 6.53 & $1.73 \%$ & $92.23 \%$ \\
\hline Potamonautes niloticus & 0.00 & 2.20 & 0.00 & 0.00 & 0.55 & $0.15 \%$ & $7.77 \%$ \\
\hline Subtotal & 15.00 & 13.30 & 0.00 & 0.00 & 7.08 & $1.88 \%$ & $100.00 \%$ \\
\hline \multicolumn{8}{|l|}{ Insecta } \\
\hline Chironomus Larvae & 50.00 & 195.70 & 62.10 & 122.00 & 107.45 & $28.51 \%$ & $97.31 \%$ \\
\hline Damselfly & 7.50 & 2.20 & 0.00 & 0.00 & 2.43 & $0.64 \%$ & $2.20 \%$ \\
\hline Caddis fly & 0.00 & 0.00 & 2.20 & 0.00 & 0.55 & $0.15 \%$ & $0.50 \%$ \\
\hline Subtotal & 57.50 & 197.90 & 64.30 & 122.00 & 110.43 & $29.30 \%$ & $100.00 \%$ \\
\hline Total & 195.50 & 446.60 & 399.50 & 466.20 & 376.83 & $100.00 \%$ & \\
\hline
\end{tabular}


The highest average of population density $\left(708 \mathrm{ind} / \mathrm{m}^{2}\right)$ was estimated at station 2, while the lowest $\left(88.75 \mathrm{ind} / \mathrm{m}^{2}\right)$ was estimated at station 7 (Table 15$)$. This result may be attributed to the nature of the bottom sediments and availability of food supply as it was considered the most significant factors determining the macrobenthos distribution (Iskaros and El-Dardir, 2010), although, Nkwoji et al. (2010) found that low macrobenthos community abundance and diversity was greatly affected by stress imposed by land based pollutants.

Seasonally, the population density of the macrobenthic fauna showed two peaks; $\left(466.2 \mathrm{ind} / \mathrm{m}^{2}\right)$ during autumn and $\left(446.6 \mathrm{ind} / \mathrm{m}^{2}\right)$ during spring due to the intensive number of Annelida and the dominance of mollusks, respectively (Fig. 4). SlavevskaStamenkovć et al. (2010) attributed same result to the high DO concentrations and freshly produced organic matter that cause flourishing of benthic community especially Oligochaeta and Diptra. On the other hand, winter showed the lowest population density with an average of $195.00 \mathrm{ind} / \mathrm{m}^{2}$. Similar results were recorded by Mageed (2000) and Fishar and Williams (2006).

Table 15: Average numbers of total benthic invertebrates groups (individual $/ \mathrm{m}^{2}$ ) recorded in the different stations in Ismailia Canal during 2010.

\begin{tabular}{|c|c|c|c|c|c|c|c|c|c|c|c|c|}
\hline Macrobenthos & $\begin{array}{l}\text { Av. } \\
\text { St1 }\end{array}$ & $\begin{array}{l}\text { Av. } \\
\text { St2 }\end{array}$ & $\begin{array}{l}\text { Av. } \\
\text { St3 }\end{array}$ & $\begin{array}{l}\text { Av. } \\
\text { St4 }\end{array}$ & $\begin{array}{l}\text { Av. } \\
\text { St5 }\end{array}$ & $\begin{array}{c}\text { Av. } \\
\text { St6 }\end{array}$ & $\begin{array}{l}\text { Av. } \\
\text { St7 }\end{array}$ & $\begin{array}{l}\text { Av. } \\
\text { St8 }\end{array}$ & $\begin{array}{l}\text { Av. } \\
\text { St9 }\end{array}$ & $\begin{array}{c}\text { Av. } \\
\text { St10 }\end{array}$ & Average & $\%$ \\
\hline Mollusca & 324.25 & 260.75 & 59.50 & 176.00 & 16.50 & 270.00 & 38.75 & 45.75 & 83.00 & 52.00 & 132.65 & $35.20 \%$ \\
\hline Annelida & 80 & 151.5 & 211 & 204.75 & 94.5 & 111.25 & 44.5 & 50 & 222.25 & 97 & 126.68 & $33.62 \%$ \\
\hline Arthopoda & 78.5 & 295.75 & 277.75 & 299.25 & 16.5 & 55.5 & 5.5 & 11 & 27.75 & 107.5 & 117.5 & $31.18 \%$ \\
\hline Total & 482.75 & 708.00 & 548.25 & 680.00 & 127.50 & 436.75 & 88.75 & 106.75 & 333.00 & 256.50 & 376.83 & \\
\hline
\end{tabular}

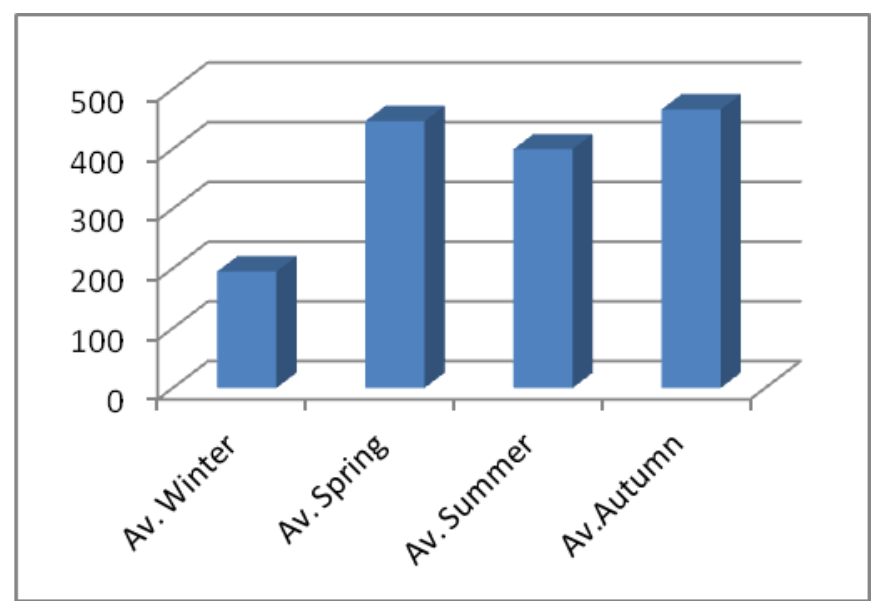

Fig. 4: Seasonal distribution of total benthic invertebrates in Ismailia Canal during 2010.

\section{Species Composition of Mollusca:}

Phylum Mollusca was the most dominant benthic macroinvertebrates during the period of investigation. It contributed about $35.20 \%$ of total Macrobenthic invertebrates, with an annual average of $132.65 \mathrm{ind} / \mathrm{m}^{2}$. The dominance of Mollusca is attributed to the flourishing of planktonic organisms which are the main food item for these grazing organisms (Fishar, 2002). During the present study, ten molluscan species were recorded, namely; Melanoides tuberculata, Cleopatra bulimoides, Bellamya unicolor, Corbicula consorbina, Lymnaea truncatula, Caelatura prasidens, Theodoxus niloticus, Bulinus truncatus, Lanistes carinatus and Physa acuta. Station 1 exhibited the most populated station, while station 5 showed the least population with an average of $324.25 \mathrm{ind} / \mathrm{m}^{2}$ and $16.5 \mathrm{ind} / \mathrm{m}^{2}$, respectively (Fig. 5). The high density 
of molluscs was attributed to the amount of silt while the poorest values were in relation to the instability of the substrate (Iskaros and El-Dardir, 2010). On the other hand, spring exhibited the maximum population density $\left(168.7 \mathrm{ind} / \mathrm{m}^{2}\right)$ due to the dominance of Melanoides tuberculata and Lymnaea truncatula. This result is mainly attributed to the predominance of phytoplankton (Fishar, 2002 ; Iskaros and El-Dardir, 2010). However, winter showed the least population density $\left(82.5 \mathrm{ind} / \mathrm{m}^{2}\right)$ due to the absence of Bellamya unicolor, Lanistes carinatus, Lymnaea truncatula, Physa acuta and Bulinus truncatus (Fig. 6).

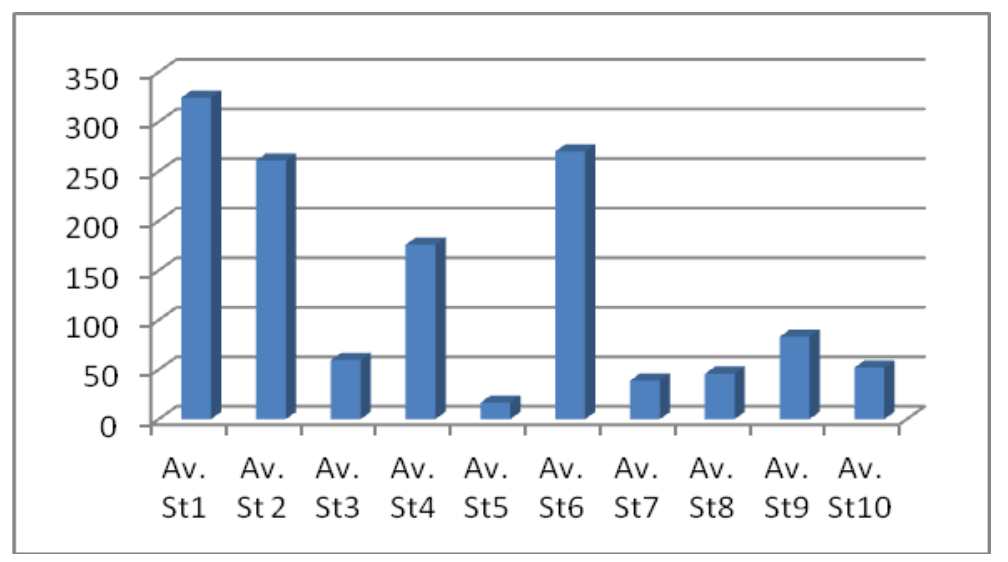

Fig. 5: Distribution of molluscs in the different stations of Ismailia Canal during 2010.

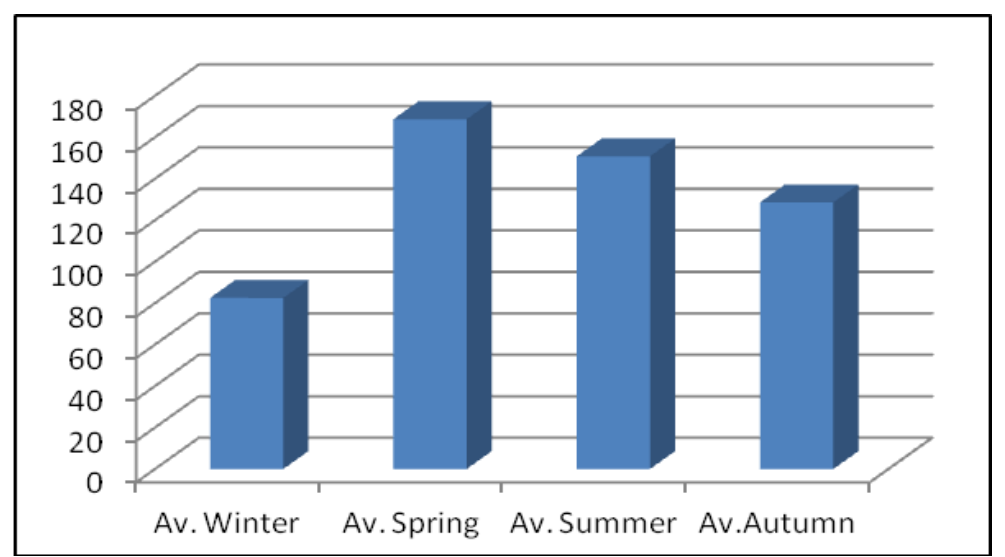

Fig. 6: Seasonal variation of Mollusca in Ismailia Canal during 2010.

Melanoides tuberculata was the most dominant molluscan species. It contributed $50.62 \%$ of total Mollusca and $17.82 \%$ of total benthic invertebrates with an average annual population density of $67.15 \mathrm{ind} / \mathrm{m}^{2}$. The distribution of this species showed a remarkable increase at station 6 with an annual average $227.75 \mathrm{ind} / \mathrm{m}^{2}$. This may be attributed to high organic matter, BOD and $\mathrm{pH}$ values as a result of the petroleum companies' effluents. On the other hand, the lowest density was at station 5 with an annual average $5.5 \mathrm{ind} / \mathrm{m}^{2}$. This may be attributed to the highest $\mathrm{Mg}, \mathrm{Cr}$ and Ba values recorded at station 5 during the present study. In addition, the structure of the sediment represents a main factor for Melanoides tuberculata distribution, where Fishar and Abdel-Regal (1998) recorded Melanoides tuberculata in sandy sediment with lowest percent of clay particles. Melanoides tuberculata was at its maximum density during spring with an average of $88.9 \mathrm{ind} / \mathrm{m}^{2}$, while, the lowest was during winter with an average of $20 \mathrm{ind} / \mathrm{m}^{2}$. This may be attributed to the distribution of phytoplankton and increasing the amount of calcium carbonate (Iskaros and El Dardir, 2010). 


\section{Species Composition of Annelida:}

Annelida considered the second dominant group among the benthic invertebrates found in the studied area. It contributed $33.62 \%$ of total benthic invertebrates with an annual average of $126.68 \mathrm{ind} / \mathrm{m}^{2}$. Iskaros and El-Dardir (2010) reported that the predominance of oligochaetes in Lake Nasser was due to their ability to adapt to various habitats and to their tolerance to low oxygen content or anoxic conditions. During the present study, annelids were represented by three species, namely, Limnodrilus hoffmeisteri, Branchiura sowerbyi and Helobdella conifera. Fig (7) shows species composition of Annelida in the investigated area. The tubificid species Limnodrilus hoffmeisteri represented the most dominant component of Annelida contributing $95.18 \%$ of total Annelida population density and $32.00 \%$ of total benthic invertebrates with an annual average density of $120.58 \mathrm{ind} / \mathrm{m}^{2}$. Similar results were reported by Iskaros and El-Dardir (2010) in Lake Nasser and Wissa (2012) in El-Rahawey region. This result is highly attributed to the ability of the tubificid species Limnodrilus sp. to resist high pollution (Wissa, 2012). As shown in Fig. (8), station 9 was the most populated station with an annual average of 222.25 ind $/ \mathrm{m}^{2}$, while station 7 was the least with an annual average of $44.5 \mathrm{ind} / \mathrm{m}^{2}$.

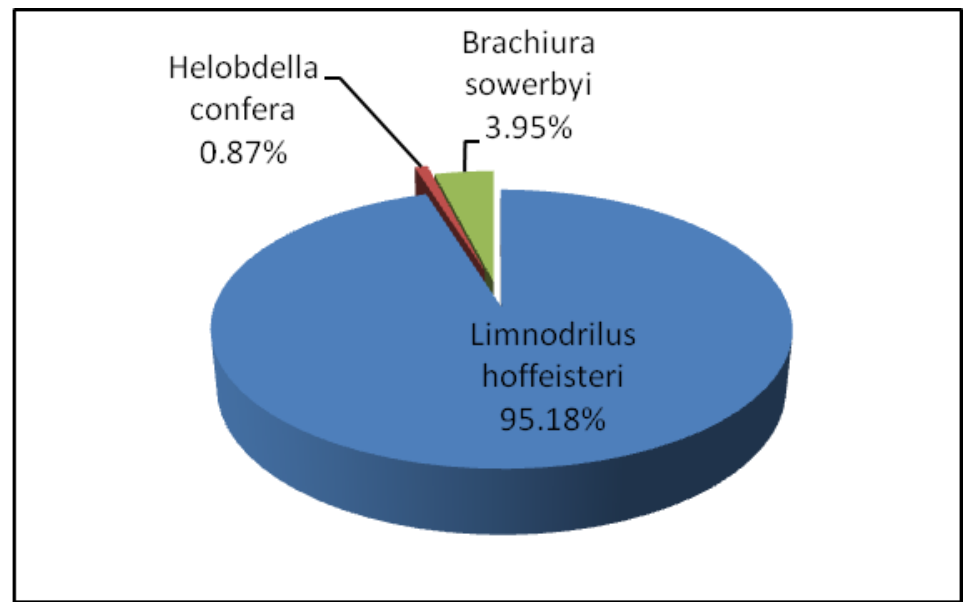

Fig. 7: Species composition of Annelida in Ismailia Canal during 2010.

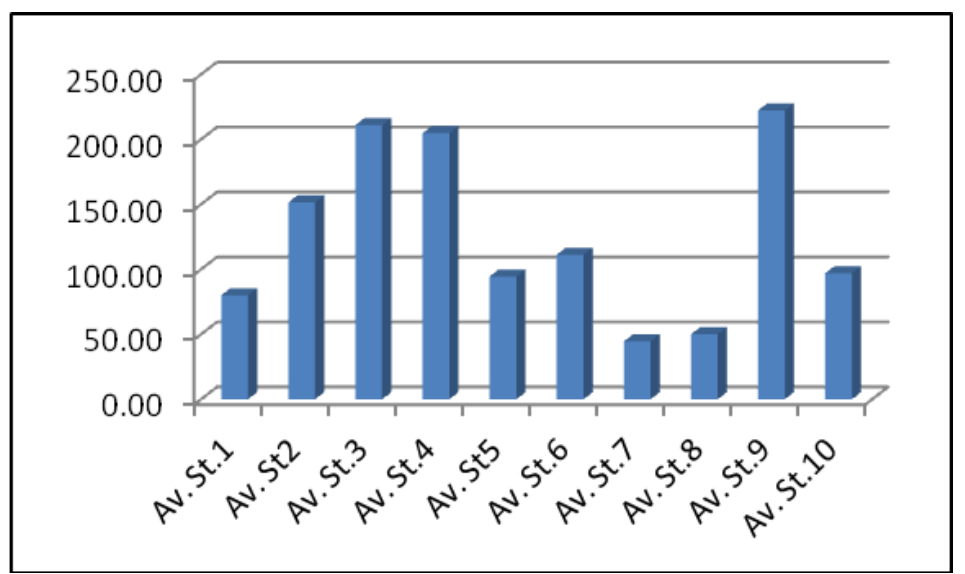

Fig. 8: Distribution of Annelida in the different stations of Ismailia Canal during 2010.

This result is mainly attributed to the dominance of Limnodrilus hoffmeisteri at station 9, which is related to the abundance of decayed macrophytes and high concentrations of nutrients and organic matter discharged from Abu Zaabal Fertilizer Company that enhances the abundance of Limnodrilus hoffmeisteri (Iskaros et al., 
2010; Slavevska-Stamenkovic' et al., 2010; Wissa, 2012). On the other hand, autumn was the most populated season with an average of $215.6 \mathrm{ind} / \mathrm{m}^{2}$, which might be attributed to the increased phytoplankton decaying during autumn that lead to increase the organic carbon, resulting in increased annelid population density (Iskaros et al., 2010), while, winter was the least with an average of $40 \mathrm{ind} / \mathrm{m}^{2}$ (Fig. 9). This may be attributed to the strong water currents which lead to unstable conditions with highest BOD values which is unfavorable habitat. It was noticeable that Limnodrilus hoffmeisteri was represented in the four seasons because it is considered as pollution tolerant species. Hence, the variation of water temperature is not the determining factor for their seasonal distribution (Wissa, 2012).

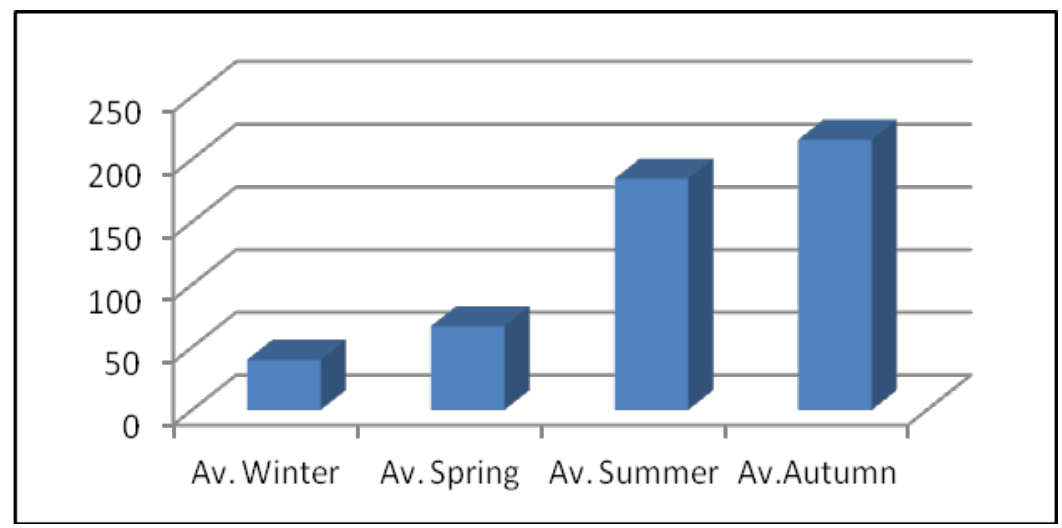

Fig. 9: Seasonal variation of Annelida in Ismailia Canal during 2010.

\section{Species Composition of Arthropoda:}

Arthropoda is the third dominant benthic group found in the studied area. It contributed about $31.18 \%$ of total benthic invertebrates with an annual average of $117.50 \mathrm{ind} / \mathrm{m}^{2}$. It was represented by two classes; Insecta and Crustacea. Insecta constituted the main component of Arthopoda (93.98\%), while Crustacea was sporadically appeared. These results are in accordance with those of Wissa (2012). The aquatic insects constituted $29.30 \%$ of total macrobenthic population density. It was represented by three insect larvae, namely, Chironomus Larvae, Damselflies and Caddis flies (Fig. 10). The larvae of Chironomidae represented the most dominant component of Insecta.

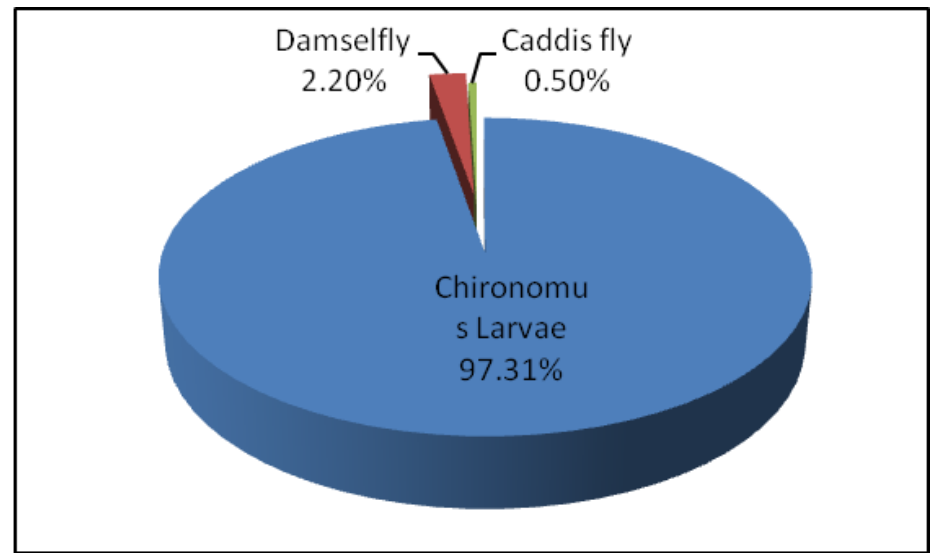

Fig. 10: Species composition of Insecta in Ismailia Canal during 2010. 
This result is in accordance with that reported by Ahmad et al. (2002), Wissa (2002) and Wissa (2012). Considering the distribution of the aquatic insects in all stations, Chironomus larvae formed $97.31 \%$ of total insect density and $28.51 \%$ of total benthic invertebrates with an annual average of $107.45 \mathrm{ind} / \mathrm{m}^{2}$. It is classified among order Diptera. These larvae inhabit littoral zones of both oligotrophic and eutrophic lakes. The increase of Chironomids is often an indication of polluted conditions (Wissa, 2002). During the present survey, the maximum population density was recorded at station 3 with an annual average density of $272.25 \mathrm{ind} / \mathrm{m}^{2}$, while station 7 was the lowest with an annual average density of $5.5 \mathrm{ind} / \mathrm{m}^{2}$. According to Ahmad et al. (2002), the abundance and dominance of Chironomidae was especially related with the amount of detritus. The highest population density was recorded during spring (195.7 ind $/ \mathrm{m}^{2}$ ). On the other hand, the lowest was during winter (50 ind $/ \mathrm{m}^{2}$ ) (Fig. 11). This result can be explained by the rise in temperature accelerate the cycle of chironomids leading to adult stage (Wissa, 2002).

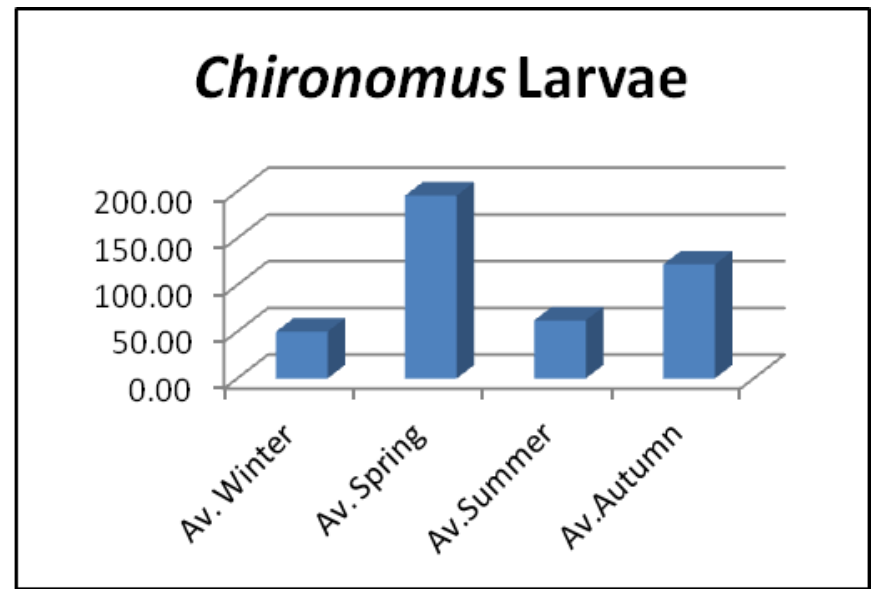

Fig. 11: Seasonal distribution of Chironomus Larvae in Ismailia Canal during 2010.

Crustacea was the least recorded group. It contributed about $1.88 \%$ of total benthic invertebrates with an annual average $7.08 \mathrm{ind} / \mathrm{m}^{2}$. The highest population density was at station $2\left(37.5 \mathrm{ind} / \mathrm{m}^{2}\right)$. Concerning seasonal variation, Crustacea was recorded only during winter and spring with higher population density during winter (15 ind $/ \mathrm{m}^{2}$ ). Crustacea was represented only by 2 species; Cardina nilotica and Potamonautes niloticus (Fig. 12). Cardina nilotica was the most dominant crustacean species. It was represented by $92.23 \%$ of total crustacean population density and $1.73 \%$ of total benthic invertebrates' density with an annual average density of 6.53 ind $/ \mathrm{m}^{2}$.

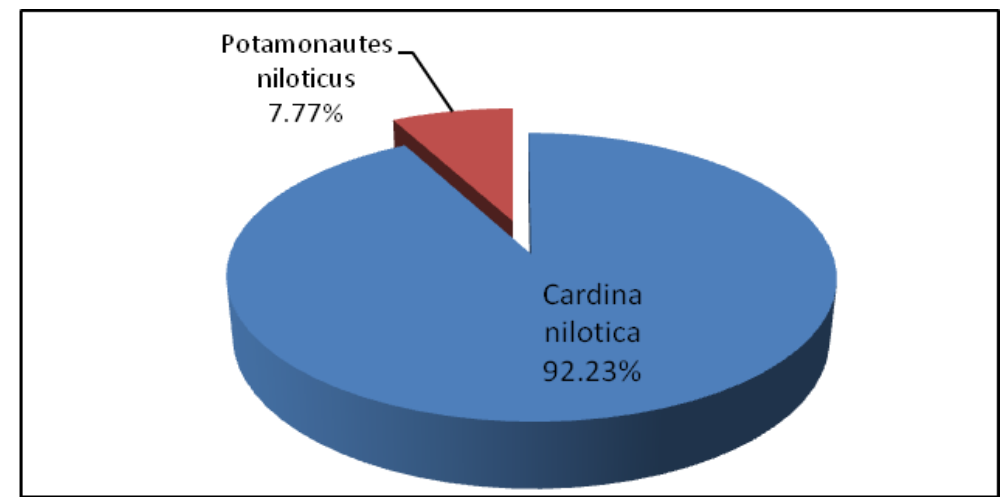

Fig. 12: Species composition of Crustacea in Ismailia Canal during 2010. 
During the present study, it was recorded only at station 2 and station 4 . The maximum population density was at station 2 with an annual average density of 37.5 ind $/ \mathrm{m}^{2}$. These results indicate that stations 2 and 4 are somewhat clear, which permits the diversity of benthic species. It was recorded only during winter and spring with a peak during winter $\left(15 \mathrm{ind} / \mathrm{m}^{2}\right)$, while it disappeared during summer and autumn. These results were supported by Wissa (2012). On the other hand, Potamonautes niloticus was represented only by $7.77 \%$ of total crustacean density and $0.15 \%$ of total benthic invertebrates. It was recorded only at station 4 with an annual average 5.5 ind $/ \mathrm{m}^{2}$.

\section{Macrobenthos diversity:}

Biodiversity provides important functions to the aquatic ecosystem due to the function done by different species in the community. Therefore, the decreased species number is considered as loss of biodiversity in polluted ecosystems that leads to habitat destruction. During the present study, the biodiversity of macrobenthos revealed that station 2 recorded the highest species richness and Shannon Weaver index value ( $d=1.8$ and $\left.\mathrm{H}^{\prime}=1.9\right)$. It could be attributed to the dominance of molluscan species (9 molluscan species) and exclusively dominance of Cardina nilotica. This result reflects the good water condition at station 2. On contrary, station 5 recorded the least species richness and Shannon Weaver index value $\left(d=0.6\right.$ and $\left.H^{\prime}=0.8\right)$ (Table 16).

Table 16: Species diversity of benthic macroinvertebrate in different stations during the period of the study.

\begin{tabular}{|c|c|c|c|c|c|c|c|c|}
\hline & S & N & d & $\mathbf{J}^{\prime}$ & Brillouin & Fisher & $\begin{array}{c}\mathbf{H}^{\prime} \\
\text { (loge) }\end{array}$ & $\begin{array}{c}\text { 1- } \\
\text { Lambda' }\end{array}$ \\
\hline St. 1 & 10 & 483 & 1.456 & 0.7731 & 1.742 & 1.784 & 1.78 & 0.7863 \\
\hline St. 2 & 13 & 708 & 1.829 & 0.7499 & 1.868 & 2.261 & 1.923 & 0.7987 \\
\hline St. 3 & 8 & 548 & 1.11 & 0.5574 & 1.128 & 1.328 & 1.159 & 0.6031 \\
\hline St. 4 & 12 & 680 & 1.687 & 0.6639 & 1.61 & 2.07 & 1.65 & 0.7394 \\
\hline St. 5 & 4 & 128 & 0.6188 & 0.6013 & 0.8234 & 0.7848 & 0.8336 & 0.428 \\
\hline St. 6 & 7 & 437 & 0.9869 & 0.7319 & 1.385 & 1.184 & 1.424 & 0.6697 \\
\hline St. 7 & 4 & 89 & 0.6688 & 0.7637 & 1.013 & 0.8611 & 1.059 & 0.6074 \\
\hline St. 8 & 7 & 107 & 1.285 & 0.8169 & 1.468 & 1.68 & 1.59 & 0.7305 \\
\hline St. 9 & 6 & 333 & 0.8609 & 0.6009 & 1.039 & 1.039 & 1.077 & 0.5217 \\
\hline St. 10 & 5 & 257 & 0.7211 & 0.7511 & 1.165 & 0.8806 & 1.209 & 0.6677 \\
\hline
\end{tabular}

Macrobenthic fauna is considered a good indicator for the biological and environmental status of the aquatic ecosystem. To examine the associations between the population density and the stations, Primer Similarity Index was applied to the data and was represented as dendrogram in Fig. (13). It is clear that stations 1 and 6 exhibited the highest similarity value. This could be attributed to the species composition of both stations are quite similar (Table 17), although species richness are different but the diversity index are quite similar, which might be attributed to the nature of the substrate in each station. Furthermore, stations 2 and 4 were similar which could be attributed to the dominance of crustacean species only in these stations. 
Table 17: Species number of different macrobenthos groups in the ten stations during 2010.

\begin{tabular}{|c|c|c|c|c|c|}
\hline Station & Mollusca & Annelida & Insecta & Crustacae & Total \\
\hline St. 1 & 7 & 2 & 1 & 0 & 10 \\
\hline St. 2 & 9 & 1 & 2 & 1 & 13 \\
\hline St. 3 & 5 & 1 & 2 & 0 & 8 \\
\hline St. $\mathbf{4}$ & 6 & 3 & 1 & 2 & 12 \\
\hline St. 5 & 2 & 1 & 1 & 0 & 4 \\
\hline St. 6 & 4 & 2 & 1 & 0 & 7 \\
\hline St. 7 & 2 & 1 & 1 & 0 & 4 \\
\hline St. 8 & 5 & 1 & 1 & 0 & 7 \\
\hline St. 9 & 4 & 1 & 1 & 0 & 6 \\
\hline St. 10 & 2 & 1 & 2 & 0 & 5 \\
\hline
\end{tabular}

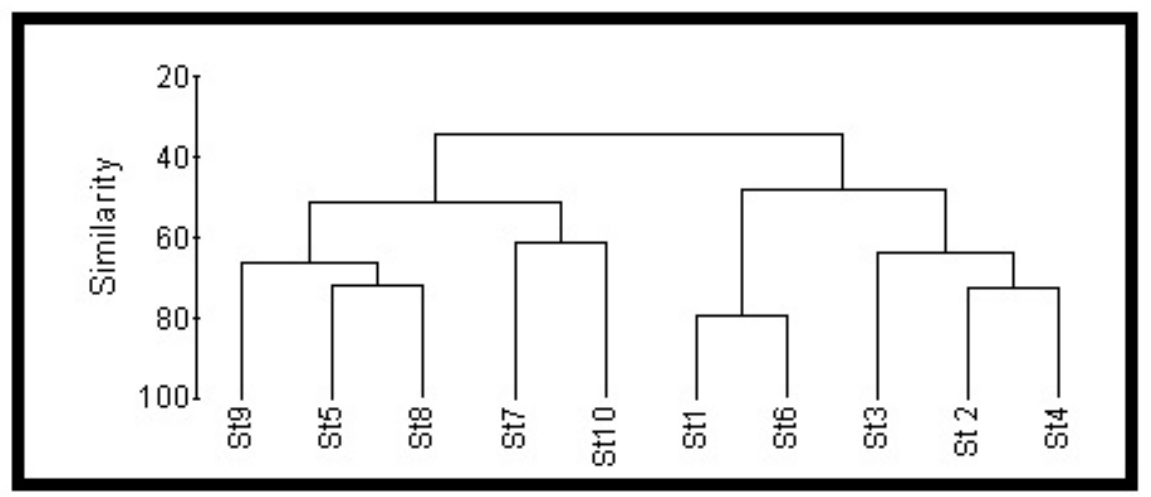

Fig. 13: Dendrogram showing similarity among the 10 studied stations in Ismailia Canal according to macrobenthic community structure.

\section{Conclusion and recommendations:}

From the previous mentioned discussion, we can conclude that:

1- Studying of water quality parameters along Ismailia Canal revealed that the discharging points of Petroleum Company and Abu Zaabal Company are the main sources of pollution in Ismailia Canal.

2- Study of the macrobenthic community in Ismailia Canal revealed that pollutants have affected their biodiversity.

\section{Recommendations:}

* Special attention should be given to mitigate pollution from these sources as their effects may become significant on the long term.

* Constant ecological and hydrological monitoring of Ismailia Canal water is needed to record any alteration in the quality and outbreak of health disorders.

* Discharging of industrial effluents into the canal has to be limited.

* Applying treatment techniques before discharging the waste water will lead to a better remediation of this important ecosystem.

\section{REFERENCES}

Abdel-Satar, A. M. (1998). Distribution of some chemical elements in River Nile environments at Great Cairo Region. Ph. D. Thesis, Faculty of Science, Cairo Univ. 
Abdel-Satar, A.M. and Elewa, A.A. (2001). Water quality and environmental assessments of the River Nile at Rossetta Branch, The Second International Conference and Exhibition for Life and Environment, 3-5 April: 136-164.

Abdel-Satar, A.M. (2005). Water quality assessment of River Nile from Idfo to Cairo. Egyptian Journal of Aquatic Research., 3 (2):200-223.

Abdo, M. H. (1998). Some environmental studies on the River Nile and Ismailia Canal in front of the industrial area of Shoubra El-Kheima. M. Sc. Thesis, Fac. of Sci., Ain Shams Univ., Cairo, Egypt.

Abdo, M. H.; Sabae, S. Z.; Haroon, B. M.; Refaat, B. M. and Mohammad, A. S. (2010). Physico-Chemical Characteristics, Microbiological Assessment and Antibiotic Susceptibility of Pathogenic Bacteria of Ismailia Canal water, River Nile, Eygpt. Journal of American Science. 6(5): 234-250.

Abdo, M.H. and El-Nasharty, S.M. (2010). Physico-Chemical Evaluations And Trace Metals Distribution in Water-Surficial Sediment of Ismailia Canal, Egypt. Nature and Science 8(5): 198-206.

Ahmad, A.; Maimon, A.; Othman, M. S. and Mohd, P. A. (2002). The potential of local benthic macroinvertebrates as a biological monitoring tool for river water quality assessment. Proceedings of the Regional Symposium on Environment and Natural Resources. 1: 464-471.

American Public Health Association, American Water Works Association, Water Environment Federation. Standard Methods for the Examination of Water and Wastewater, $21^{\text {th }}$ ed. American Public Health Association, Washington, DC. 2005.

Barbour, M.T.; Gerritsen, J.; Snyder, B. D. and Stribling, J. B. (1999). Rapid Bioassessment Protocols for Use in Streams and Wadeable Rivers:Periphyton, Benthic Macroinvertebrates and Fish . 2nd Edition . EPA 841-B-99-002. U.S. Environmental Protection Agency. Offi ce of Water . Washington, District of Columbia, U.S.A . 339 pp.

Brinkhurst, R. O. (1971). A guide for identification of British aquatic oligochaetae. Freshwater Biological Association, Scientific Publication number 22. 55pp.

Brown, D. S. (1980). Freshwater snails of Africa and their medical importance. Taylor and Francis. London, 487pp.

Chapman, D. (1992). Water Quality Assessments: A guide to the Use of Biota, Sediments and Water in Environmental Monitoring. Chapman \& Hall, London, (on behalf of UNESCO/WHO/UNEP). pp., 585.

Elewa, A. A.; Saad, E. A.; Shehata, M.B. and Ghallab, M. H. (2007). Studies on the effects on the water quality of Lake Manzala, Egypt. Egypt. J. Aquat. Biol. \& Fish., vol. 11 (2): 65- 78.

El-Haddad, E.S.M. (2005). Some Environmental Studies on Water and Sediment of Ismailia Canal from El Mazalat to Anshas Region. M.Sc. Thesis, Faculty of Science (Girl Branch) Al Azhar University.

El-Shimy, N. A. (1994). A contribution to some leech fauna (Hirudinea) in the Middle East. J. Egyptian German Society of Zoology., 14: 13-27.

Fishar, M. R. and Abdel-Regal, R. M. (1998). Macrobenthic invertebrates in relation to sediment properties in some fish farms. Egypt. J. Aquat. Biol. \& Fish., Vol. 2(2): 87- 100.

Fishar, M. R. A. (1999). Distribution and abundance of benthic assemblages in ElGamil basin (Lake Manzala) A-Meiobenthos, Bull. Nat. Int. Oceanogr, Fish, A. R. E, 25: 151 - 165. 
Fishar, M. R. A. (2002). Structure, composition and distribution of benthic macroinvertebrates in E-Salam Canal, Egypt. Egypt. J. Aquat. Biol. \& Fish., Vol. 6(1): 83-93.

Fishar M. R. and Williams, W. P. (2006). A feasibility study to monitor the macroinvertebrate diversity of the River Nile using three sampling methods. Hydrobiologia 556:137-147.

Fishar, M.R.; Thorne, R. and Williams, W.P. (2006). Physico-chemical conditions and macroinvertebrate fauna in the River Nile from Aswan to Cairo. African Journal of Aquatic science, 31 (2):247-259.

Habashy, M. M. (1993). Taxonomical and ecological studies of aquatic insects in rearing and nursing ponds fish farms, Ain Shams University.

Hassan, M. M. (2008). Ph. D. Thesis, Ain Shams Univ.

Ibrahim, A. M.; Bishai, H. M. and Khalil, M. T. (1999). Freshwater mollusca of Egypt. Egyptian Environmental Affair Agency, publication of national biodiversity, unit number 10 .

Ibrahim, S. M.; Shallof, K. A. and Salama, H. M. (2008). Effect of Environmental Conditions of Abu-Zabal Lake on Some Biological, Histological and Quality Aspects of Fish. Global Veterinaria, 2(5): 257-270.

Iskaros, I. A. and El-Dardir, M. (2010). Factors affecting the distribution and abundance of bottom fauna in Lake Nasser, Egypt. Nature and Science, 8(7): 95-108.

Iskaros, I. A.; Gindy, N. N. and El-Dardir, M. (2010). Long - term fluctuations of macrobenthic invertebrates in Aswan Water Reservoir, Egypt. International Journal of Environmental Science and Engineering (IJESE)., 1: 37-48

Mageed, A. A. (2000). Meroplanktonic stages of benthic invertebrates in Lake Qaroun (El-Fayoum- Egypt). Egypt. J. Aquat. Biol. \& Fish., 4 (2): 27- 42.

Mohammed, A. S. (2008). Microbiological studies on Ismailia Canal, River Nile, Egypt. M.Sc. Thesis, Faculty of Science. Al Azhar University.

Nkwoji, J. A.; Yakub, A.; Ajani, G. E.; Balogun, K. J.; Renner, K. O.; Igbo, J. K.; Ariyo, A. A. and Bello, B. O. (2010). Seasonal variations in the water chemistry and benthic macroinvertebrates of a south western lagoon, Lagos, Nigeria. Journal of American Science, 6 (3): 85-92.

Rabeh, S.A. (2000). Thermal and microbial pollution in the River Nile at industrial region of Shoubra El-Kheima, Egypt. Egyptian Academy Society for Environmental Development, 1(1): 83-98.

Ruffo, M. (1982). The Amphipoda of the Mediterranean. Mem. Del. Inst. Oceano., Monaco. Part I: Gammaridae.

Saad, M.A.H. (1973). A limnological studies on Nasser Lake and the Nile in Egypt, Water supply and Management, 4: 8192.

Saad, M.A.H. and Abdel-Moati, M. A. (1997). Nutrient Salts in the Damietta Estuary of the Nile, an Area under Stress, Journal of Conference Abstracts, 2(2): BIOGEOMON, 97.

Sabae, S.Z. and Abdel-Satar, A.M. (2001). Chemical and Bacteriological studies on El-Salam Canal, Egypt, J. Egypt. Acad. Soc. Environ. Develop., 2(1): 173-197.

Sabae, S.Z.; Hazaa, M.M.; Aballah, S.A.; Awny, N. and Dabbor, S.M. (2006): Studies on bacterial indicators of water pollution and bioremediater isolates for $\mathrm{Cu} 2+$, Fe2+ and Zn2+ in Rosetta Brach River Nile, Egypt. Egyptian Journal of Biotechnology, 22: 77-104.

Shama, S. A.; Goher, M. E.; Abdo, M. H. ; Kaial, S. M. and Ahmed, A. A. (2011). Physico-chemical characteristics and heavy metal contets in water of Wadi 
El-Rayan Lakes, Western desert, Egypt. Egypt. J. Aquat. Biol. And Fish., 15(2): 225- 240.

Slavevska-Stamenkovic', V.; Smiljkov, S.; Prelic', D.; Paunovic', M.; Atanackovic', A. and Rimcheska, B. (2010). Structural characteristic of benthic macroinvertebrate in the Mantovo Reservoir (South-East Part of the $\mathrm{R}$. Macedonia). BALWOIS 2010-Ohrid, Republic of Macedonia.

Toufeek, M. A. F. and Korium, M. A. (2009). Physicochemical Characteristics of Water Quality in Lake Nasser Water. Global J. Envir. Res., 3 (3): 141-148.

Wissa, J. B. (2002). Ecological Studies on Benthic Invertebrates of Nile River at North Cairo Region. M. Sc. Thesis, Fac. Sci., Cairo Univ., 179 pp.

Wissa, J. B. (2012). Ecological Studies on Benthic Invertebrates as Indicators of Organic Pollution in El-Rahawey Region, Giza, at River Nile, Egypt. Ph.D. Thesis, Fac. Sci., Ain Shams Univ., 249 pp.

World Health Organization (WHO), (1993). Guidelines for drinking water quality, $2^{\text {nd }}$ ed., vol. 1. Recommendations. World Health Organization. Geneva.

Wrona, E.J. and Cash, K. J. (1996). The ecosystem approach to environmental assessment: moving from theory practice. Aqu. Ecosys., 5:89-97.

Yousry, K.M. (2009). Assessment and Modeling of Water Quality and Zooplankton of El-Salam Canal, Egypt. Ph. D. Thesis, Dep. Environmental Basic Sciences. Institute of Environmental Studies and Research. Ain Shams University.

\section{ARABIC SUMMARY}

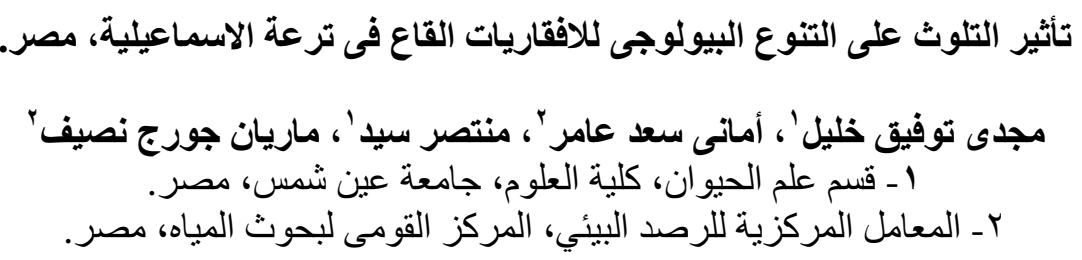

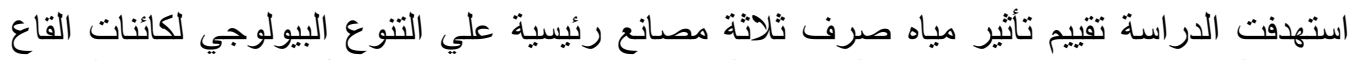

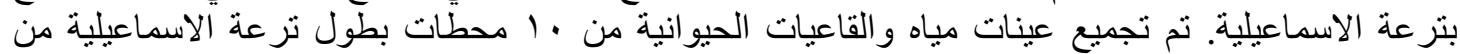

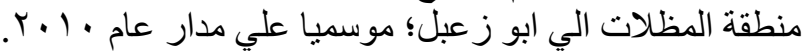
أوضحت النتائج ان معظم قيم نوعية المياه في نطاق المعدل المسموح به به عاله عالميا، في حين ان ان منطقة

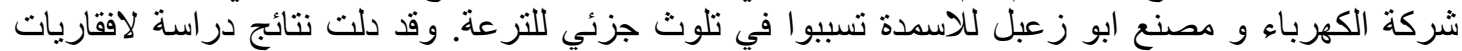

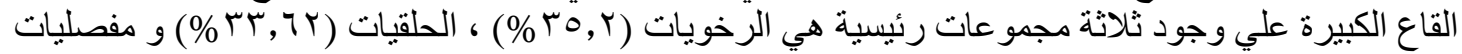

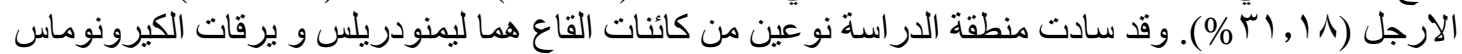

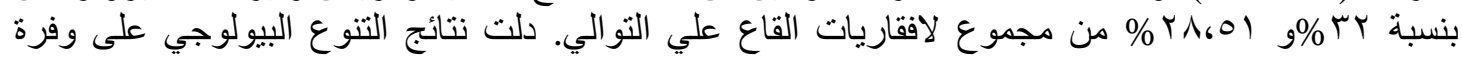

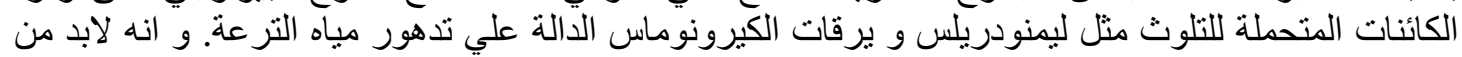

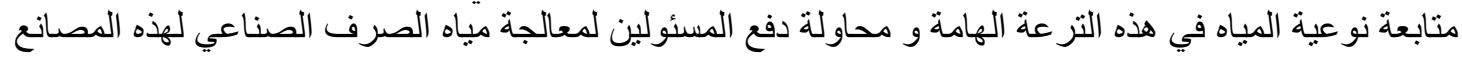
قبل ان تصب في مياه التر عة. 